\title{
Oligarchies in spatial environments
}

Citation for published version (APA):

Ehlers, L. H., \& Storcken, A. J. A. (2007). Oligarchies in spatial environments. METEOR, Maastricht University School of Business and Economics. METEOR Research Memorandum No. 042 https://doi.org/10.26481/umamet.2007042

Document status and date:

Published: 01/01/2007

DOI:

10.26481/umamet.2007042

Document Version:

Publisher's PDF, also known as Version of record

\section{Please check the document version of this publication:}

- A submitted manuscript is the version of the article upon submission and before peer-review. There can be important differences between the submitted version and the official published version of record.

People interested in the research are advised to contact the author for the final version of the publication, or visit the DOI to the publisher's website.

- The final author version and the galley proof are versions of the publication after peer review.

- The final published version features the final layout of the paper including the volume, issue and page numbers.

Link to publication

\footnotetext{
General rights rights.

- You may freely distribute the URL identifying the publication in the public portal. please follow below link for the End User Agreement:

www.umlib.nl/taverne-license

Take down policy

If you believe that this document breaches copyright please contact us at:

repository@maastrichtuniversity.nl

providing details and we will investigate your claim.
}

Copyright and moral rights for the publications made accessible in the public portal are retained by the authors and/or other copyright owners and it is a condition of accessing publications that users recognise and abide by the legal requirements associated with these

- Users may download and print one copy of any publication from the public portal for the purpose of private study or research.

- You may not further distribute the material or use it for any profit-making activity or commercial gain

If the publication is distributed under the terms of Article $25 \mathrm{fa}$ of the Dutch Copyright Act, indicated by the "Taverne" license above, 
Lars Ehlers, Ton Storcken

Oligarchies in Spatial Environments

$\mathrm{RM} / 07 / 042$

JEL code: D70, D71

\section{METE@R}

Maastricht research school of Economics of TEchnology and ORganizations

Universiteit Maastricht

Faculty of Economics and Business Administration P.O. Box 616

NL - 6200 MD Maastricht

phone : ++31433883830

fax : ++31433884873 


\title{
Oligarchies in Spatial Environments*
}

\author{
Lars Ehlers ${ }^{\dagger}$ and Ton Storcken ${ }^{\ddagger}$
}

September 2007

\begin{abstract}
In spatial environments we consider social welfare functions satisfying Arrow's requirements, i.e. weak Pareto and independence of irrelevant alternatives. Individual preferences measure distances between alternatives according to the $L_{p}$-norm (for a fixed $p \geq 1$ ). When the policy space is multi-dimensional and the set of alternatives has a non-empty interior and it is compact and convex, any quasi-transitive welfare function must be oligarchic. As a corollary we obtain that for transitive welfare functions weak Pareto, independence of irrelevant alternatives, and non-dictatorship are inconsistent if the set of alternatives has a non-empty interior and it is compact and convex.

Journal of Economic Literature Classification Numbers: D70, D71.
\end{abstract}

\section{Introduction}

A social welfare function is a procedure for aggregating profiles of individual preferences into social orderings. Arrow's theorem shows that it is impossible for a social

*We acknowledge useful comments and suggestions by John Duggan, William Thomson, and John Weymark.

†Département de Sciences Économiques and CIREQ, Université de Montréal, Montréal, Québec H3C 3J7, Canada; e-mail: lars.ehlers@umontreal.ca

${ }_{\ddagger}^{\ddagger}$ Department of Quantitative Economics, Maastricht University, P.O. Box 616, 6200 MD Maastricht, The Netherlands; e-mail: t.storcken@ke.unimaas.nl 
welfare function to satisfy weak Pareto (if all individuals strictly prefer one alternative to another, then so does society), independence of irrelevant alternatives (the social ranking of two alternatives only depends on the individual rankings of these alternatives), and non-dictatorship when the preference domain is unrestricted. When the set of alternatives is structured, the assumption of unrestricted domain might be unreasonable. One important exception of this kind are spatial environments. In spatial environments, alternatives are points in a multi-dimensional space of issue positions and individual preferences are continuous, quasi-concave, and have bliss points. ${ }^{1}$

There is one well-known spatial environment in which Arrow's requirements are consistent. If the number of voters is odd and the policy space is one-dimensional, then simple majority voting is transitive and satisfies weak Pareto, independence of irrelevant alternatives, and anonymity (Black ,1948; Arrow, 1951, 1963). Simple majority voting is an example of an Arrovian welfare function belonging to the following class: if the number of voters is $n$, fix $n-1$ preferences of additional constant voters, and apply to each profile of individual preferences majority voting over this profile and the $n-1$ fixed voters. ${ }^{2,3}$

If the policy space is multi-dimensional and unbounded, and preferences are Euclidean, then Arrow's requirements are inconsistent (Border, 1984). Other proofs show that the domain of Euclidean preferences is "saturating" and apply a result due to Kalai, Muller, and Satterthwaite (1979) to deduce Arrow's impossibility (Le Breton and Weymark, 1996, 2000, 2002; Campbell, 1993 ${ }^{4}$ ). However, the proofs of

\footnotetext{
${ }^{1}$ An excellent review of the literature is Le Breton and Weymark (2000).

${ }^{2}$ Ehlers and Storcken (2002) provide a characterization of all welfare functions satisfying weak Pareto and independence of irrelevant alternatives.

${ }^{3}$ For finite sets of alternatives Sethuraman, Teo, and Vohra $(2003,2006)$ introduce an integer linear programming formulation of anonymous and transitive Arrovian social welfare functions (ruling out social indifferences). They show that any such welfare function is monotonic if and only if it is simple majority voting with $n+1$ fixed voters for any domain with no Condorcet triples and which contains an ordering and its inversion.

${ }^{4}$ Campbell (1993) drops weak Pareto and imposes instead continuity of social preference.
} 
these results rely importantly on the unboundedness of the policy space. As far as we know, Arrow's theorem has not been established in spatial environments when the set of alternatives is compact and convex. It is natural to assume that a government faces budget constraints and therefore the set of policy alternatives becomes bounded. We derive Arrow's theorem in such spatial environments. If individual preferences are measure distances according to a given $L_{p}$-norm and the set of alternatives has a non-empty interior and it is compact and convex, then weak Pareto, independence of irrelevant alternatives, and non-dictatorship are inconsistent, i.e. again decisiveness of a coalition spreads from one pair of alternatives to all pairs of alternatives and dictatorship results.

Instead of showing Arrow's theorem directly, we will establish a stronger result. Gibbard (1969) and Mas-Colell and Sonnenschein (1972) require social orderings only to be quasi-transitive and show that for a finite set of alternatives with an unrestricted domain any quasi-transitive welfare functions satisfying weak Pareto and independence of irrelevant alternatives must be oligarchic. This means that there exists a non-empty coalition $S$ (called the oligarchy) such that an alternative $a$ is socially strictly preferred to another alternative $b$ if and only if all members of the oligarchy agree that $a$ is strictly preferred to $b$. Our main result shows the following: if individual preferences individual preferences are measure distance according to the $L_{p}$-norm and the set of alternatives has a non-empty interior and it is compact and convex, then any quasi-transitive welfare function satisfying weak Pareto and independence of irrelevant alternatives must be oligarchic.

The paper is organized as follows. In Section 2 we introduce our notation and the main definitions. In Section 3 we state that weak Pareto, independence of irrelevant alternatives, and non-dictatorship are inconsistent if the policy space is compact, convex, and at least two-dimensional. In Section 4 we introduce oligarchic welfare functions and give our main result: any quasi-transitive welfare function satisfying weak Pareto and independence of irrelevant alternatives must be oligarchic. Section 
5 contains the proof of our main result. Section 6 concludes.

\section{Notation and Definitions}

We use the same terminology and notation as Le Breton and Weymark (2000). Let $\mathbb{R}$ denote the set of real numbers and $\mathbb{R}^{m}$ denote the $m$-dimensional Euclidean space. Let $N \equiv\{1,2, \ldots, n\}$ denote a finite set of agents with $n \geq 2$, and let $A \subseteq \mathbb{R}^{m}$ denote a set of alternatives. Each point in $\mathbb{R}^{m}$ identifies the changes in the level of $m$ different policies, for example public spending on police, health care, and so on.

Let $\mathcal{W}$ denote the set of all complete, reflexive, and transitive relations over $A$. An element of $\mathcal{W}$ is called a weak ordering over $A$. Let $\mathcal{Q}$ denote the set of all complete, reflexive, and quasi-transitive relations over $A$. An element of $\mathcal{Q}$ is called a quasi ordering over $A$.

Given $R_{i} \in \mathcal{Q}$, the corresponding strict relation, $P_{i}$, and the indifference relation, $I_{i}$, are defined as follows: for all $a, b \in A$, (i) $a P_{i} b \Leftrightarrow \neg b R_{i} a$, and (ii) $a I_{i} b \Leftrightarrow a R_{i} b$ and $b R_{i} a$. Note that if $\neg a R_{i} b$, then by completeness of $R_{i}, a R_{i} b$. Hence, $\neg b R_{i} a$ is enough to describe the strict relation $P_{i}$. Recall that $R_{i}$ is transitive if for all $a, b, c \in A$, $a R_{i} b$ and $b R_{i} c \Rightarrow a R_{i} c$; and $R_{i}$ is quasi-transitive if for all $a, b, c \in A, \neg b R_{i} a$ and $\neg c R_{i} b \Rightarrow \neg c R_{i} a$.

Let $\mathcal{R} \subseteq \mathcal{W}$ denote an individual's preference domain. A (preference) profile is a list $R \equiv\left(R_{i}\right)_{i \in N} \in \mathcal{R}^{N}$. A (social) welfare function assigns to each profile a quasi ordering over $A$. Any welfare function $f$ is a mapping $f: \mathcal{R}^{N} \rightarrow \mathcal{Q}$ such that for all $R \in \mathcal{R}^{N}, f(R) \in \mathcal{Q}$. We call $f(R)$ the social ordering (that $f$ assigns to $R$ ). As $f(R)$ is quasi-transitive for all $R \in \mathcal{R}^{N}$ welfare function $f$ is said to be quasi-transitive.

We call a welfare function $f$ transitive if it is a mapping $f: \mathcal{R}^{N} \rightarrow \mathcal{W}$ such that for all $R \in \mathcal{R}^{N}, f(R) \in \mathcal{W}$

Note that social orderings need not belong to the individual preference domain $\mathcal{R}$. Other authors impose the restriction that any social ordering belongs to each individual's preference domain (for example, Peters, van der Stel, and Storcken, 1992, 
and Bossert and Weymark, 1993).

Arrow's requirements are as follows. The first axiom says that if all agents strictly prefer $a$ to $b$, then $a$ should also be socially strictly preferred to $b$.

Weak Pareto: For all $R \in \mathcal{R}^{N}$ and all $a, b \in A$, if for all $i \in N, a P_{i} b$, then $\neg b f(R) a$.

Given $R \in \mathcal{R}^{N}, X \subseteq A$, and $j \in N$, let $R_{j} \mid X$ denote the restriction of $R_{j}$ to $X$, and $R \mid X \equiv\left(R_{i} \mid X\right)_{i \in N}$. The second axiom says that the social ordering of two alternatives only depends on the profile of individual preferences restricted to these two alternatives.

Independence of Irrelevant Alternatives: For all $R, \bar{R} \in \mathcal{R}^{N}$ and all $a, b \in A$, if $R|\{a, b\}=\bar{R}|\{a, b\}$, then $f(R)|\{a, b\}=f(\bar{R})|\{a, b\}$.

A welfare function is Arrovian if it satisfies weak Pareto and independence of irrelevant alternatives. A welfare function is dictatorial if there exists some agent such that for each profile the social strict preference relation respects the strict preference relation of this agent.

Non-Dictatorship: There exists no $i \in N$ such that for all $R \in \mathcal{R}^{N}$ and all $a, b \in A$, if $a P_{i} b$, then $\neg b f(R) a$.

Arrow's Theorem shows that for transitive welfare functions, weak Pareto, independence of irrelevant alternatives, and non-dictatorship are incompatible if the set of alternatives is finite and the individual preference domain $\mathcal{R}$ contains all strict orderings over the set of alternatives. 


\section{Arrow's Theorem for Multi-Dimensional Policy Spaces}

Throughout we consider environments where the set of alternatives is multi-dimensional and connected with respect to the standard topology on $\mathbb{R}^{m}$. Let $A \subseteq \mathbb{R}^{m}$ be a set of alternatives such that the relative interior of $A$ in $\mathbb{R}^{m}$ is non-empty. A preference relation $R_{i} \in \mathcal{W}$ is $L_{p}$-single-peaked if there exists a bliss point $p\left(R_{i}\right) \in A$ such that for all $a, b \in A, a R_{i} b \Leftrightarrow\left\|a-p\left(R_{i}\right)\right\|_{p} \leq\left\|b-p\left(R_{i}\right)\right\|_{p}$ (here $\|\cdot\|_{p}$ denotes the $L_{p}$ norm in $\mathbb{R}^{m}$ for a given $\left.p \geqslant 1\right)^{5}$. In case $p=2$ the $L_{2}$-single-peaked preferences are also called Euclidean. Let $\mathcal{R}_{p}$ denote the set of all $L_{p}$-single-peaked preferences over $A$. Note that $p$ remains fixed throughout.

For the special case of $A=\mathbb{R}^{m}$ and the Euclidean norm, Border (1984) and Le Breton and Weymark (1996) establish Arrow's theorem for transitive welfare functions. For $A=\mathbb{R}_{+}^{m}$, Le Breton and Weymark (2000, 2002) show Arrow's theorem for transitive welfare functions. However, all these results rely importantly on the unboundedness of the set of alternatives. In real life, policy makers face budget constraints and these results do not apply.

A corollary of our main result will be the following theorem: Arrow's requirements are inconsistent if the set of alternatives has a non-empty and connected interior and it is contained in the closure of its interior (i.e. its boundary has no "tails").

Let $\operatorname{int}(A)$ denote the interior of $A$ in $\mathbb{R}^{m}$ and $\operatorname{cl}(A)$ the closure of $A$ in $\mathbb{R}^{m}$.

Theorem 1 Let $A \subseteq \mathbb{R}^{m}$ be such that $\operatorname{int}(A) \neq \emptyset$, int $(A)$ is connected, and $A \subseteq$ $\operatorname{cl}(\operatorname{int}(A))$. If $m \geq 2$, then for transitive welfare functions weak Pareto, independence of irrelevant alternatives, and non-dictatorship are inconsistent on the domain $\mathcal{R}_{p}^{N}$.

In particular, Theorem 1 yields Arrow's theorem for compact and convex sets of policy alternatives with non-empty interior.

\footnotetext{
${ }^{5}$ For any $x \in \mathbb{R}^{m}$ we have $\|x\|_{p}=\left(x_{1}^{p}+x_{2}^{p}+\cdots+x_{m}^{p}\right)^{1 / p}$
} 
If the interior of the set of alternatives is non-empty and not connected, then an Arrovian social welfare function may not be dictatorial.

Example 1 Let $m \equiv 2, p \equiv 2, A_{1} \equiv[0,1] \times[0,1], A_{2} \equiv[0,1] \times[9,10]$, and $A \equiv$ $A_{1} \cup A_{2}$. For all $R \in \mathcal{R}_{2}^{N}$, let $f(R)\left|A_{1}=R_{1}\right| A_{1}, f(R)\left|A_{2}=R_{2}\right| A_{2}$, and for all $a \in A_{1}$ and all $b \in A_{2}, f(R)\left|\{a, b\}=R_{1}\right|\{a, b\}$. Then $f$ is a transitive Arrovian welfare function satisfying non-dictatorship.

The conclusion of Example 1 does not remain true if the sets $A_{1}$ and $A_{2}$ are connected through a line segment, for example if $A=A_{1} \cup A_{2} \cup[(0,0),(0,10)]$. For this set of alternatives an Arrovian welfare function must be dictatorial. ${ }^{6}$

If the boundary of $A$ contains tails, then again an Arrovian welfare function may not be dictatorial.

Example 2 Let $m \equiv 2, p \equiv 2, N \equiv\{1,2\}, B \equiv[0,1] \times[0,1]$, and $L \equiv](0,1),(0,2)]$. Let $A \equiv B \cup L$. For all $R \in \mathcal{R}_{2}^{N}$ and all $a, b \in A$, (i) if $a, b \in L, a_{2}<b_{2}$, and for some $i \in N$, $a P_{i} b$, then $\neg b f(R) a$, and (ii) otherwise, $f(R)\left|\{a, b\}=R_{1}\right|\{a, b\}$. We show that $f$ is a transitive welfare function.

Claim: For all $R \in \mathcal{R}_{2}^{N}, f(R)$ is transitive.

Proof of Claim. If $p\left(R_{1}\right) \in B$, then $f(R)=R_{1}$. Let $p\left(R_{1}\right) \in L$ and $a, b, c \in A$. If $a, b, c \in B$ or $a, b, c \in L$, then it is easy to check that $f(R) \mid\{a, b, c\}$ is transitive. If $a \in L$ and $b, c \in B$, then $f(R)\left|\{a, b, c\}=R_{1}\right|\{a, b, c\}$ and $f(R) \mid\{a, b, c\}$ is transitive. Let $a, b \in L$ and $c \in B$. Then $f(R)\left|\{a, c\}=R_{1}\right|\{a, c\}$ and $f(R) \mid\{b, c\}=$ $R_{1} \mid\{b, c\}$. Obviously, if $f(R)\left|\{a, b\}=R_{1}\right|\{a, b\}$, then $f(R) \mid\{a, b, c\}$ is transitive. Let $f(R)\left|\{a, b\} \neq R_{1}\right|\{a, b\}$. Without loss of generality, suppose that $a_{2}<b_{2}$. Thus, by (i), $\neg b f(R) a$, and $a_{2} \leq p_{2}\left(R_{1}\right)$. Then $a P_{1} c$ and $\neg c f(R) a$. Hence, $f(R) \mid\{a, b, c\}$ is transitive.

\footnotetext{
${ }^{6}$ By Theorem $2, f \mid A_{1}$ and $f \mid A_{2}$ are dictatorial. Because $A_{1}$ and $A_{2}$ are connected through the line segment $[(0,0),(0,10)]$ it follows from Theorem 1 that the dictator of $f \mid A_{1}$ and $f \mid A_{2}$ is the same and $f$ is dictatorial.
} 
Now it is easy to check that $f$ is a transitive welfare function satisfying weak Pareto, independence of irrelevant alternatives, and non-dictatorship.

\section{Oligarchies}

Above any welfare function satisfied transitivity. Below social orderings are only required to be quasi-transitive. In order to formulate our main result, we need to introduce the notion of decisiveness and oligarchies.

Given $\emptyset \neq S \subseteq N$ and $a, b \in A$, we say that $S$ is decisive over " $a$ preferred to $b$ " if for all $R \in \mathcal{R}_{p}^{N}$ we have $\neg b f(R) a$ whenever $a P_{i} b$ for all $i \in S$. Let $D(a, b)$ denote the set of all coalitions that are decisive over " $a$ preferred to $b$ ". We say that $S$ blocks " $a$ preferred to $b$ " if for all $R \in \mathcal{R}_{p}^{N}$ we have $b f(R) a$ whenever $b P_{i} a$ for all $i \in S$. Let $B(a, b)$ denote the set of all coalitions that block " $a$ preferred to $b$ ".

Let $f$ be a welfare function and $S$ be a non-empty coalition. Then $f$ is called oligarchic with oligarchy $S$ if for all $a, b \in A$ such that $a \neq b$ we have $S \in D(a, b)$ and $\{i\} \in B(a, b)$ for all $i \in S$. In other words, for oligarchic welfare functions $a$ is socially strictly preferred to $b$ if and only if all members of the oligarchy strictly prefer $a$ to $b$. Note that $f$ is dictatorial if there exists $i \in N$ such that $f$ is oligarchic with oligarchy $\{i\}$. It is easy to check that oligarchic welfare functions satisfy weak Pareto and independence of irrelevant alternatives and that the chosen social orderings are quasi-transitive.

Our main result establishes that oligarchic welfare functions are the only quasitransitive welfare functions satisfying weak Pareto and independence of irrelevant alternatives if the set of alternatives has a non-empty and connected interior and it is contained in the closure of its interior.

Theorem 2 Let $A \subseteq \mathbb{R}^{m}$ be such that $\operatorname{int}(A) \neq \emptyset$, $\operatorname{int}(A)$ is connected, and $A \subseteq$ cl(int $(A))$. If $m \geq 2$, then oligarchic welfare functions are the only quasi-transitive welfare functions satisfying weak Pareto and independence of irrelevant alternatives 
on the domain $\mathcal{R}_{p}^{N}$.

We show Theorem 2 in the next section. Theorem 1 will be an immediate consequence of Theorem 2.

\section{Proof of Theorem 2}

Let $f$ be a welfare function satisfying weak Pareto and independence of irrelevant alternatives on the domain $\mathcal{R}_{p}^{N}$. Given $X \subseteq A$, let $f^{X}$ denote the restriction of $f$ to the set of alternatives $X$. Formally, for all $R \in \mathcal{R}_{p}^{N}$ such that for all $i \in N$, $p\left(R_{i}\right) \in X$, let $f^{X}(R \mid X) \equiv f(R) \mid X$. It is straightforward that $f^{X}$ inherits weak Pareto and independence of irrelevant alternatives from $f$.

We introduce additional notation. Let $a, b, c \in \mathbb{R}^{m}$ be three distinct alternatives. Let $[a, b]$ denote the line segment between $a$ and $b$, i.e. $[a, b] \equiv\{\lambda a+(1-\lambda) b \mid \lambda \in$ $[0,1]\}$. Let $H(a, b)$ denote the surface of points with equal distance to $a$ and $b$. This surface bisects $[a, b]$. In the non-degenerate case, when $a, b$, and $c$ are non-collinear, the surfaces $H(a, b), H(a, c)$, and $H(b, c)$ determine six disjoint open subspaces, say $\operatorname{space}(a b c)$, space $(a c b), \operatorname{space}(b a c), \operatorname{space}(b c a)$, space $(c a b)$, and space $(c b a)$ (see Figure 1 for Euclidean preferences in $\mathbb{R}^{2}$ ). We named these spaces such that for all $R_{i} \in \mathcal{R}_{p}$, $p\left(R_{i}\right) \in \operatorname{space}(a c b) \Leftrightarrow a P_{i} c P_{i} b$ and likewise for the other spaces. Note that the collective boundary of $\operatorname{space}(a b c)$ and $\operatorname{space}(a c b)$ is a subset of $H(b, c)$. Let space $(a(b c))=$ $\operatorname{space}(a b c) \cap \operatorname{space}(a c b)$ that is the set of all points $x \in H(b, c)$ such that for $R_{i} \in \mathcal{R}_{p}$, if $p\left(R_{i}\right)=x$, then $a P_{i} b I_{i} c$. Note that $H(a, b) \cap H(a, c) \cap H(b, c) \neq \emptyset$ and $\{a, b, c\}$ is a free triple in the domain $\mathcal{R}_{p}$ if $H(a, b) \cap H(a, c) \cap H(b, c) \cap \operatorname{int}(A) \neq \emptyset$. Then the domain $\mathcal{R}_{p}$ contains all conceivable rankings of $a, b$, and $c$. Let $d$ and $u$ be two points in $\mathbb{R}^{m}$ such that for all coordinates $j \in\{1,2, \ldots, m\} d_{j} \leq u_{j}$. Let $\operatorname{box}(d, u)=\{x \in$ $\mathbb{R}^{m} \mid$ for all coordinates $\left.j \in\{1,2, \ldots, m\}, d_{j} \leq x_{j} \leq u_{j}\right\}$ denote the hyper box between $d$ and $u$. Let $\operatorname{cpbox}(d, u)=\left\{x \in \mathbb{R}^{m} \mid\right.$ for all coordinates $j \in\{1,2, \ldots, m\}, d_{j}=x_{j}$ or $\left.x_{j}=u_{j}\right\}$ denote the set of corner points of $\operatorname{box}(d, u)$. Now for every triple of these 
corner points, say $a, b$ and $c, m \in H(a, b) \cap H(a, c) \cap H(b, c)$, where $m=\frac{1}{2} d+\frac{1}{2} u$ the middle of $[d, u]$. If $\operatorname{box}(d, u) \subseteq \operatorname{int}(A)$, then any triple of its corner points is a free triple.

Given $a \in \mathbb{R}^{m}$ and $\epsilon>0$, let $\odot(a, \epsilon)$ denote the open ball with center $a$ and radius $\epsilon$, i.e. $\bigodot(a, \epsilon) \equiv\left\{x \in \mathbb{R}^{m} \mid\|a-x\|_{p}<\epsilon\right\}$. Given $a, b, c \in \mathbb{R}^{m}$, let plane $(a, b, c)$ denote the plane spanned by $a, b$, and $c$, i.e. plane $(a, b, c) \equiv\{a+\lambda(b-a)+\mu(c-a) \mid \lambda, \mu \in \mathbb{R}\}$. Let $v$ and $w$ be two points belonging to the boundary of $\odot(a, \epsilon)$, i.e. $\|a-v\|_{p}=\epsilon$ and $\|a-w\|_{p}=\epsilon$. The circular arc between $v$ and $w$ on $\odot(a, \epsilon)$ in plane $(a, v, w)$ is denoted by $\operatorname{arc}(a ; v, w)$.

Lemma 1 Let $a \in \operatorname{int}(A)$ and $b \in A$ such that $a \neq b$. Let $\epsilon>0$ be such that $\bigodot(a, \epsilon) \subseteq \operatorname{int}(A)$. Then there exist $c \in \bigodot(a, \epsilon)$ and $d \in \operatorname{int}(A)$ such that for all $c^{\prime} \in$ $\operatorname{arc}(d ; a, c),\left\{a, b, c^{\prime}\right\}$ is a free triple in $\mathcal{R}_{p}$.

Proof. Because $\operatorname{int}(A)$ is connected and $A \subseteq \operatorname{cl}(\operatorname{int} A)$, on a path from $a$ to $b^{\prime}$, a point arbitrarily close to $b$, we may choose $d \in H(a, b) \cap \operatorname{int}(A)$. Further, as $d \in$ $\operatorname{int}(A)$ we may choose $d$ such that it does not belong to $[a, b]$. Then $a, b$ and $d$ are not collinear. Because $d \in \operatorname{int}(A)$, we have for some $\epsilon^{\prime}>0, \bigodot\left(d, \epsilon^{\prime}\right) \subseteq \operatorname{int}(A)$. Now choose $c \in \bigodot(a, \epsilon) \cap$ plane $(a, b, d)$ such that $c$ belongs to the boundary of $\odot(d,\|a-d\|)$. For $c^{\prime} \in \operatorname{arc}(d ; c, a)$ we have that $H(a, b) \cap H\left(a, c^{\prime}\right) \cap \operatorname{plane}(a, b, d)=\{d\}$. Thus, $H(a, b) \cap H\left(a, c^{\prime}\right) \cap H\left(b, c^{\prime}\right) \cap \operatorname{plane}(a, b, d)=\{d\}$. Since $d \in \operatorname{int} A$, the two previous facts imply that for all $c^{\prime} \in \operatorname{arc}(d ; c, a),\left\{a, b, c^{\prime}\right\}$ is a free triple in $\mathcal{R}_{p}$.

The following is a direct consequence of Blair and Pollack (1979).

Lemma 2 Let $a, b, c \in A$ be such that $\{a, b, c\}$ is a free triple in the domain $\mathcal{R}_{p}$. Then $f^{\{a, b, c\}}$ is oligarchic.

Now if $\{a, b, c\}$ is a free triple in the domain $\mathcal{R}_{p}$, then let $\emptyset \neq S_{\{a, b, c\}} \subseteq N$ be such that $f^{\{a, b, c\}}$ is oligarchic with oligarchy $S_{\{a, b, c\}}$. Note that any oligarchy is uniquely determined, i.e. if $f^{\{a, b, c\}}$ is oligarchic with oligarchies $S_{1}$ and $S_{2}$, then $S_{1}=S_{2}$. 
The next lemma establishes that $f$ is oligarchic on any open ball, which is contained in the interior of $A$.

Lemma 3 Let $a \in \operatorname{int}(A)$ and $\epsilon>0$ be such that $\odot(a, \epsilon) \subseteq \operatorname{int}(A)$. Then there exists $S \subseteq N$ such that,$f^{\odot(a, \epsilon)}$ is oligarchic with oligarchy $S$.

Proof. Let $d, u \in(a, \epsilon)$ be such that $d_{j} \leq u_{j}$ for all $j \in\{1, \ldots, m\}$ and $\operatorname{box}(d, u) \subseteq$ $\bigodot(a, \epsilon)$. Every triple $a^{\prime}, b, c \in \operatorname{cpbox}(d, u)$ is a free triple in $\mathcal{R}_{p}$. Thus, by Lemma 2, $f^{\left\{a^{\prime}, b, c\right\}}$ is oligarchic, say with oligarchy $S_{\left\{a^{\prime}, b, c\right\}}$. The same holds for any other triple of corner points $\left\{a^{\prime \prime}\right\}$ of box $(d, u)$. But then $S_{\left\{a^{\prime}, b, c\right\}}=S_{\left\{a^{\prime \prime}, b, c\right\}}$. Now it is immediate that $f^{\operatorname{cpbox}(d, u)}$ is oligarchic with oligarchy $S_{\left\{a^{\prime}, b, c\right\}}$. Since this is true for any hyper box in $\odot(a, \epsilon)$ and the triples of corner points of those are connected, i.e. there are sequences of triples of corner points of boxes starting with one triple and ending with another triple, whose subsequent members have at least two points in common, it follows that $f^{\odot(a, \epsilon)}$ is oligarchic with oligarchy $S_{\{a, b, c\}}$.

Lemma 4 There exists a non-empty $S \subseteq N$ such that for all $a \in \operatorname{int}(A)$ and all $b \in A$, with $a \neq b, f^{\{a, b\}}$ is oligarchic with oligarchy $S$.

Proof. First we proof the following: for all $a \in \operatorname{int}(A)$, there exists a non-empty $S_{a} \subseteq N$ such that for all $b \in A \backslash\{a\}$,

$$
f^{\{a, b\}} \text { is oligarchic with oligarchy } S_{a} \text {. }
$$

In order to proof this claim, let $a \in \operatorname{int}(A)$ and $b, b^{\prime} \in A$ be such that $a, b$ and $b^{\prime}$ are distinct. It is sufficient to prove that $f^{\{a, b\}}$ and $f^{\left\{a, b^{\prime}\right\}}$ are both oligarchic with the same oligarchy.

Lemma 1 implies that there exist $c, c^{\prime} \in \odot(a, \epsilon) \subseteq \operatorname{int}(A)$ such that $\|a-c\|=$ $\left\|a-c^{\prime}\right\|$ and both $\{a, b, c\}$ and $\left\{a, b^{\prime}, c^{\prime}\right\}$ are free triples. Thus, by Lemma 2, both $f^{\{a, b, c\}}$ and $f^{\left\{a, b^{\prime}, c^{\prime}\right\}}$, respectively, are oligarchic with oligarchy $S_{\{a, b, c\}}$ and $S_{\left\{a, b^{\prime}, c^{\prime}\right\}}$, respectively. By Lemma 3 and $a, c, c^{\prime} \in \odot(a, \epsilon), S_{\{a, b, c\}}=S_{\left\{a, b^{\prime}, c^{\prime}\right\}}$, which implies (1). 
In order to finish the proof of Lemma 4, let $a, a^{\prime} \in \operatorname{int}(A)$. By (1), it is sufficient to show $S_{a}=S_{a^{\prime}}$. If $a=a^{\prime}$, then this is trivial. If $a \neq a^{\prime}$, then by (1) we have $f^{\left\{a, a^{\prime}\right\}}$ is oligarchic with oligarchies $S_{a}$ and $S_{a^{\prime}}$. Therefore, $S_{a}=S_{a^{\prime}}$, the desired conclusion. $\square$

Theorem 2 will follow from our final lemma.

Lemma 5 There exists a non-empty $S \subseteq N$ such that $f$ is oligarchic with oligarchy $S$.

Proof. By Lemma $4, f^{\text {intA } A}$ is oligarchic, say with oligarchy $S$. Let $a, b \in A \backslash \operatorname{int}(A)$. First, we show $S \in D(a, b)$. Let $R \in \mathcal{R}_{p}^{N}$ be such that $a P_{i} b$ for all $i \in S$. Let $R^{\prime} \in \mathcal{R}_{p}^{N}$ be such that for all $i \in S, p\left(R_{i}^{\prime}\right)=a$, and for all $i \in N \backslash S, R_{i}^{\prime}=R_{i}$. Because $A \subseteq \operatorname{cl}(\operatorname{int}(A))$, there exists $c \in \operatorname{int}(A)$ such that for all $i \in S, a P_{i}^{\prime} c P_{i}^{\prime} b$. By Lemma 4, we have both $S \in D(a, c)$ and $S \in D(c, b)$. Thus, both $\neg c f\left(R^{\prime}\right) a$ and $\neg b f\left(R^{\prime}\right) c$, and by quasi-transitivity of $f, \neg b f\left(R^{\prime}\right) a$. By $R^{\prime}|\{a, b\}=R|\{a, b\}$ and independence of irrelevant alternatives, $\neg b f(R) a$. Because $R$ was arbitrary, we obtain $S \in D(a, b)$.

Second, we show $\{j\} \in B(b, a)$ for all $j \in S$. Suppose to the contrary that for some $R \in \mathcal{R}_{p}^{N}$ we have both $a P_{j} b$ and $\neg a f(R) b$. Partition $N$ as follows: $V=\left\{i \in N \mid b P_{i} a\right\}$, $U=\left\{i \in N \mid a I_{i} b\right\}$, and $T=\left\{i \in N \mid a P_{i} b\right\}$. Obviously, $j \in T$.

Because $A \subseteq \operatorname{cl}(\operatorname{int} A)$, we may choose $a^{\prime} \in \operatorname{int}(A)$ arbitrarily close to $a$ and $b^{\prime} \in$ $\operatorname{int}(A)$ arbitrarily close to $b$. Because $\operatorname{int}(A)$ is connected, there is a path $\pi:[0,1] \longrightarrow$ $\operatorname{int}(A)$ from $a^{\prime}$ to $b^{\prime}$ such that $\pi(0)=a^{\prime}$ and $\pi(1)=b^{\prime}$. Because $a^{\prime}$ is arbitrarily close to $a$ and $b^{\prime}$ is arbitrarily close to $b$, we may assume that $H(a, b)$ separates $a^{\prime}$ and $b^{\prime}$. Thus, for some $t \in[0,1] \pi(t) \in H(a, b)$. Since $\pi$ is continuous, $t_{1} \equiv \min \{t \in[0,1]$ : $\pi(t) \in H(a, b)\}$ is well-defined. Let $d \equiv \pi\left(t_{1}\right)$.

Next we show space $(a d b) \cap A \neq \emptyset$. The surface $H(a, b)$ divides $\mathbb{R}^{m}$ in two open (half-)spaces $H_{a} \equiv\left\{x \in \mathbb{R}^{m}:\|a-x\|_{p}<\|b-x\|_{p}\right\}$ and $H_{b} \equiv\left\{x \in \mathbb{R}^{m}:\|b-x\|_{p}<\right.$ $\left.\|a-x\|_{p}\right\}$. Note that $\pi(t) \in H_{a}$ for all $0 \leq t<t_{1}$. Because $a^{\prime}$ is arbitrarily close to $a$, we may assume that $H(a, d)$ separates $a^{\prime}$ and $d$. Now $\pi$ also connects $a^{\prime}$ and $d$. Thus, for some $0 \leq t_{0}<t_{1}$ we have $\pi\left(t_{0}\right) \in H(a, d) \cap H_{a}$. Since $\pi\left(t_{0}\right) \in \operatorname{int}(A)$, it follows 
that $\bigodot\left(\pi\left(t_{0}\right), \epsilon\right) \subseteq \operatorname{int}(A)$ for some $\epsilon>0$. Because $\pi\left(t_{0}\right) \in H(a, d) \cap H_{a}$ it follows that $H(b, d)$ separates $b$ and $\pi\left(t_{0}\right)$. Hence, $\odot\left(\pi\left(t_{0}\right), \epsilon\right) \cap \operatorname{space}(a d b) \neq \emptyset$.

Let $R^{\prime} \in \mathcal{R}_{p}^{N}$ be such that for all $i \in V, p\left(R_{i}^{\prime}\right) \in \operatorname{int} A$ is close to $d$ and $\left\|a-p\left(R_{i}^{\prime}\right)\right\|_{p}>\left\|b-p\left(R_{i}\right)^{\prime}\right\|_{p}$; for all $i \in U, p\left(R_{i}^{\prime}\right)=d$; and for all $i \in T, p\left(R_{i}^{\prime}\right) \in$ space $(a d b) \cap A$. Then for all $i \in V, d P_{i}^{\prime} b P_{i}^{\prime} a$; for all $i \in U, d P_{i}^{\prime} b I_{i}^{\prime} a$; and for all $i \in T, a P_{i}^{\prime} d P_{i}^{\prime} b$. By weak Pareto, $\neg b f\left(R^{\prime}\right) d$. By $R^{\prime}|\{a, b\}=R|\{a, b\}$, independence of irrelevant alternatives, and $\neg a f(R) b, \neg a f\left(R^{\prime}\right) b$. Hence, by quasi-transitivity of $f, \neg a f\left(R^{\prime}\right) d$. By $d \in \operatorname{int}(A)$ and Lemma $4, f^{\{a, d\}}$ is oligarchic with oligarchy $S$, a contradiction to $\{j\} \in B(d, a)$ and $a P_{j}^{\prime} d$.

Finally, we show Theorem 1.

Proof of Theorem 1. Obviously, if $f$ satisfies the requirements of Theorem 1, then $f$ satisfies the requirements of Theorem 2. Hence, by Theorem $2, f$ is oligarchic with some non-empty oligarchy $S$. If $|S|=1$, then $f$ is dictatorial and Theorem 1 is true.

If $|S| \geq 2$, then let $j \in S$ and $a, b, c \in \operatorname{int}(A)$ be such that $\{a, b, c\}$ is a free triple in the domain $\mathcal{R}_{p}$. Obviously, the existence of such a triple is guaranteed by $\operatorname{int}(A) \neq \emptyset$ and Lemma 1.

Then let $R \in \mathcal{R}_{p}^{N}$ be such that $a P_{j} b P_{j} c$ and $b P_{i} c P_{i} a$ for all $i \in N \backslash\{j\}$. By weak Pareto, $\neg c f(R) b$. Because $f$ is oligarchic with oligarchy $S$ and $j \in S$, we have $j \in B(a, b)$. Thus, by $a P_{j} b, a f(R) b$. Then from transitivity of $f(R)$ and $\neg c f(R) b$ we obtain $\neg c f(R) a$. Since for all $i \in N \backslash\{j\}$, we have $c P_{i} a$, this implies $\{i\} \notin B(a, c)$ for all $i \in N \backslash\{j\}$. This contradicts $|S| \geq 2$ and $f$ is oligarchic with oligarchy $S$.

\section{Conclusion}

As the careful reader may have observed, for the proof of Theorem 2 it is crucial that for any hyperbox $\operatorname{box}(d, u)$, any triple of its corner points is free in the domain $\mathcal{R}_{p}$. 
This property is also true for other domains such as $L_{p}$-single-dipped preferences: a preference relation $R_{i} \in \mathcal{W}$ is $L_{p}$-single-dipped if there exists a worst point $d\left(R_{i}\right) \in A$ such that for all $a, b \in A, a R_{i} b \Leftrightarrow\left\|a-d\left(R_{i}\right)\right\|_{p} \geq\left\|b-d\left(R_{i}\right)\right\|_{p}$. Let $\mathcal{D}_{p}$ denote the set of all $L_{p}$-single-dipped preferences. Single-dipped preferences play an important role for the location of public bads such as waste deposits or nuclear power plants. Similarly to the domain $\mathcal{R}_{p}, a, b$, and $c$ is a free triple in the domain $\mathcal{D}_{p}$ if and only if $H(a, b) \cap H(a, c) \cap H(b, c) \cap \operatorname{int}(A) \neq \emptyset$. Now the proof of Theorem 2 can be easily modified to show Theorem 2 for quasi-transitive welfare functions defined over the domain $\mathcal{D}_{p}^{N}$.

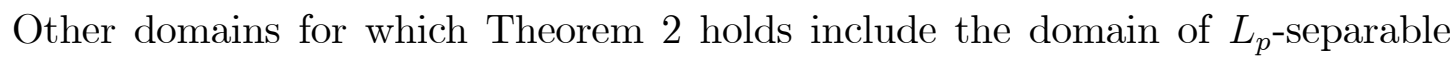
preferences: a preference relation $R_{i} \in \mathcal{W}$ is $L_{p}$-separable if there exists a bliss point $p\left(R_{i}\right) \in A$ and $\alpha_{i}>0$ for $i \in\{1, \ldots, m\}$, such that for all $a, b \in A, a R_{i} b \Leftrightarrow$ $\sum_{i=1}^{m} \alpha_{i}\left|a_{i}-p\left(R_{i}\right)_{i}\right|^{p} \leq \sum_{i=1} \alpha_{i}\left|b_{i}-p\left(R_{i}\right)_{i}\right|^{p}$. Let $\mathcal{R}_{s p}$ denote the set of all $L_{p^{-}}$separable preferences. For $L_{p}$-separable preferences, Theorem 2 can be either shown directly by modifying its proof or via showing that $L_{p}$-separable preferences are isomorphic to $L_{p^{-}}$ single-peaked preferences. This means there is a continuous bijective transformation $\varphi$ on $\mathbb{R}^{m}$ such that

$$
\left(\sum_{i=1}^{m} \alpha_{i}\left|a_{i}-b_{i}\right|^{p}\right)^{1 / p}=\|\varphi(a)-\varphi(b)\|_{p}
$$

for all $a, b \in \mathbb{R}^{m}$. The function $\varphi$ can be simply defined by by $\varphi_{i}(a)=\alpha_{i}^{1 / p} a_{i}$ for all $i \in\{1, \ldots, m\}$. Then Theorems 1 and 2 can be rephrased for this class of isomorphic distance functions because weak Pareto and independence of irrelevant alternatives are preserved by bijective transformations.

\section{References}

Arrow, K.J., 1951, Social Choice and Individual Values, John Wiley Press, New York.

Arrow, K.J. (1959): "Rational Choice Functions and Orderings," Economica 26:121- 
127.

Arrow, K.J., 1963, Social Choice and Individual Values, 2nd ed., John Wiley Press, New York.

Black, D. (1948): "On the Rationale of Group Decision Making," Journal of Political Economy 56:23-34.

Blair, D.H., and R.A. Pollak (1979): "Collective Rationality and Dictatorship: The Scope of the Arrow Theorem," Journal of Economic Theory 21:186-194.

Border, K.C. (1984): "An Impossibility Theorem for Spatial Models," Public Choice 43:293-305.

Bossert, W. and J.A. Weymark (1993): "Generalized Median Social Welfare Functions," Social Choice and Welfare 10:17-33.

Campbell, D.E. (1993): "Euclidean Individual Preference and Continuous Social Preference," European Journal of Political Economy 9, 541-550.

Ehlers, L., and T. Storcken (2002): "Arrow's Theorem in Spatial Environments," Cahier 2002-03, Department of Economics, Université de Montréal, http://www.sceco.umontreal.ca/publications/etext/2002-03.pdf.

Gibbard, A. (1969): "Social Choice and Arrow's Conditions," Working Paper.

Kalai, E., E. Muller, and M.A. Satterthwaite (1979): "Social Welfare Functions when Preferences are Convex, Strictly Monotonic, and Continuous," Public Choice 34:87-97.

Le Breton, M. and J.A. Weymark, 1996, An Introduction to Arrovian Social Welfare Functions on Economic and Political Domains, in "Social Choice and Political Economy" (Norman Schofield ed.), Kluwer, Dordrecht/Norwell, MA, 25-61. 
Le Breton, M. and J.A. Weymark, 2000, Arrovian Social Choice Theory on Economic Domains, Handbook of Social Choice and Welfare, K.J. Arrow, A.K. Sen, and K. Suzumura ed., North-Holland, forthcoming.

Le Breton, M. and J.A. Weymark (2002): "Social Choice with Analytic Preferences," Social Choice and Welfare 19:637-657.

Mas-Colell, A., and H. Sonnenschein (1972): "General Possibility Theorems for Group Decision Functions," Review of Economic Studies 39:185-192.

Peters, H., H. van der Stel, and T. Storcken (1992): "Independence of Irrelevant Alternatives and Strategy-Proofness on Economic Domains," in Game Theory and Economic Applications, Proceedings, New Delhi, India, December 1990, B. Dutta, D. Mookherjee, T. Parthasarathy, T.E.S. Ragahavan, D.Ray, and S.Tijs (ed.), Lecture Notes in Economics and Mathematical Systems Springer-Verlag Berlin Heidelberg 1992.

Sethuraman, J., C.P. Teo, and R.V. Vohra (2003): "Integer Programming and Arrovian Social Welfare Functions," Mathematics of Operations Research 28:309-326.

Sethuraman, J., C.P. Teo, and R.V. Vohra (2006): "Anonymous Monotonic Social Welfare Functions," Journal of Economic Theory 128:232-254. 


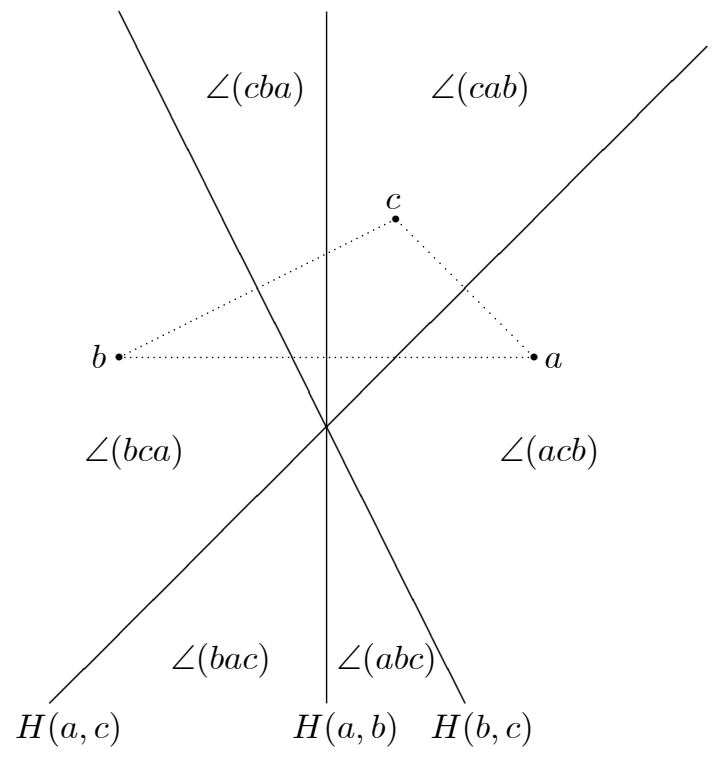

Figure 1: Illustration of the sets $\angle(a b c), \angle(a c b), \angle(b a c), \angle(b c a), \angle(c a b)$, and $\angle(c b a)$ for non-colinear $a, b$, and $c$ in $\mathbb{R}^{2}$. 